Pure Appl. Chem., Vol. 75, No. 1, pp. 39-46, 2003.

(C) 2003 IUPAC

\title{
Applications of tert-butanesulfinamide in the asymmetric synthesis of amines*
}

\author{
Jonathan A. Ellman \\ Center for New Directions in Organic Synthesis, Department of Chemistry, \\ University of California at Berkeley, Berkeley, CA 94720, USA
}

\begin{abstract}
Butanesulfinamide is prepared using catalytic enantioselective methods in two steps from the extremely inexpensive oil waste by-product, tert-butyl disulfide. Direct condensation of tert-butanesulfinamide with aldehydes and ketones provides tert-butanesulfinyl imines in uniformly high yields. The tert-butanesulfinyl group activates the imines for the addition of many different classes of nucleophiles, serves as a powerful chiral directing group, and after nucleophilic addition is readily cleaved by treatment with acid. A wide range of highly enantioenriched amines, including $\alpha$-branched and $\alpha, \alpha$-dibranched amines, $\alpha$ - and $\beta$-amino acids, 1,2- and 1,3-amino alcohols and $\alpha$-trifluoromethyl amines are efficiently synthesized using this methodology.
\end{abstract}

\section{INTRODUCTION}

Numerous pharmaceutical agents, natural products, and synthetic materials contain chiral amine functionality. For this reason, the asymmetric synthesis of amines is of fundamental importance to many synthesis applications. Over the past several years, we have developed a method for the asymmetric synthesis of a broad range of amines using the extremely versatile chiral amine reagent, tert-butanesulfinamide (Fig. 1) [1,2]. Direct condensation of tert-butanesulfinamide with aldehydes and ketones provides tert-butanesulfinyl imines in high yields. A variety of transformations, such as additions of nucleophiles, may then be performed upon the sulfinyl imines with high diastereoselectivity and in high yields to provide the desired amine products after cleavage of the sulfinyl group.

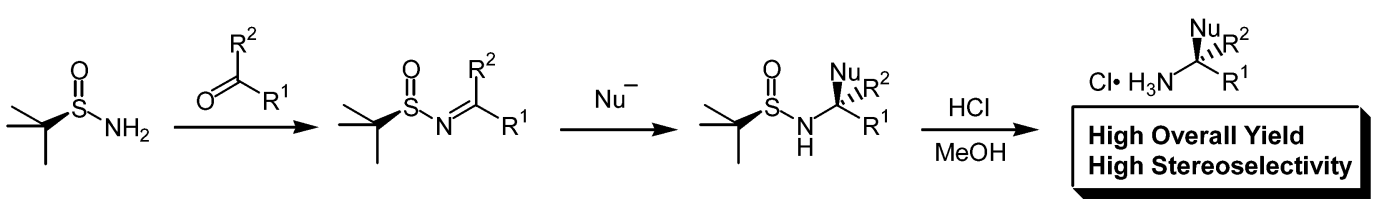

Fig. 1 Asymmetric synthesis of amines using tert-butanesulfinamide.

\section{ASYMMETRIC SYNTHESIS OF tert-BUTANESULFINAMIDE}

The development of a practical and highly efficient method to prepare enantiomerically pure tert-butanesulfinamide was important for the general use of this reagent. The most practical approach is the two-step process of catalytic asymmetric oxidation of tert-butyl disulfide followed by reaction of

*Lecture presented at the $14^{\text {th }}$ International Conference on Organic Synthesis (ICOS-14), Christchurch, New Zealand, 14-18 July 2002. Other presentations are published in this issue, pp. 1-70. 
the resulting tert-butanethiosulfinate intermediate with an amide anion (Fig. 2) [3]. tert-Butyl disulfide is an oil waste by-product and therefore serves as an extremely inexpensive starting material. Oxidation of tert-butyl disulfide can be accomplished with good conversion and in 89-91\% ee using $\mathrm{H}_{2} \mathrm{O}_{2}$ as an inexpensive stoichiometric oxidant, $\mathrm{CHCl}_{3}$ as solvent, and with only $0.25 \mathrm{~mol} \%$ of $\mathrm{VO}(\mathrm{acac})_{2}$ and chiral ligand (derived from tert-butylglycinol). Because the reaction is performed in air at a high concentration $(1.5 \mathrm{M})$, the reaction can easily be carried out on one mole scale in any standard laboratory setting. We have recently developed even more practical conditions. First, acetone, which provides a completely homogeneous reaction solution, is used as a solvent owing to its lower cost and minimal toxicity $\left(\mathrm{H}_{2} \mathrm{O}_{2}\right.$ must be added slowly to maintain high enantioselectivity). Second, the chiral ligand has been modified such that it is prepared from cis-1,2-aminoindanol, for which both enantiomers are available commercially at modest cost. Addition of lithium amide in ammonia to the tert-butanethiosulfinate intermediate provides tert-butanesulfinamide with complete inversion at sulfur. After a single crystallization, enantiomerically pure material is obtained in $68 \%$ overall yield from tert-butyl disulfide.
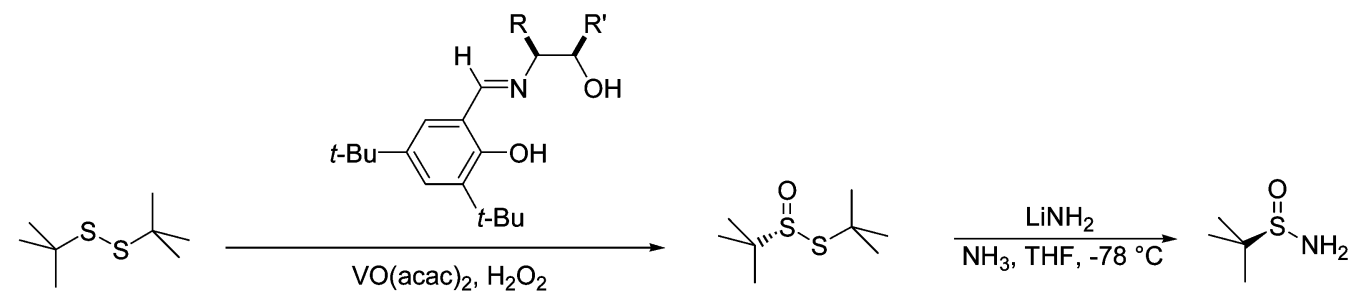

Fig. 2 Preparation of enantiomerically pure tert-butanesulfinamide.

\section{PREPARATION OF tert-BUTANESULFINYL IMINES}

High-yielding and general methods for the preparation of tert-butanesulfinyl imines are critical to the successful application of tert-butanesulfinamide in the asymmetric synthesis of amines. We developed the first direct condensation of sulfinamides with aldehydes and ketones to provide sulfinyl aldimines and ketimines, respectively [4,5]. The most straightforward method for the preparation of tert-butanesulfinyl aldimines is the condensation of aldehydes and tert-butanesulfinamide with $\mathrm{CuSO}_{4}$ as a stoichiometric water scavenger and Lewis acid catalyst (eq. 1). Simple filtration and extraction provides the sulfinyl imine in high purity. We have found $\mathrm{Ti}(\mathrm{OEt})_{4}$ to be the ideal water scavenger and Lewis acid for the preparation of tert-butanesulfinyl ketimines (eq. 2). Ti(OEt) ${ }_{4}$ is also effective for sterically hindered aldehydes such as pivaldehyde [6].

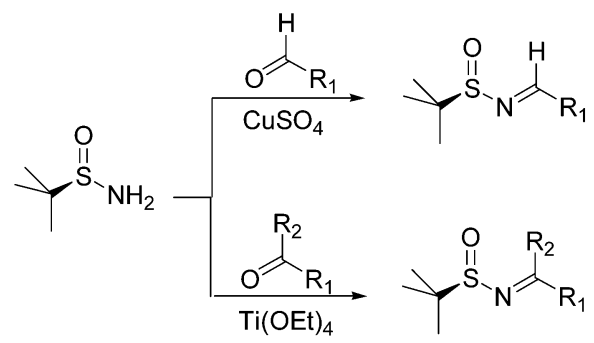

\section{NUCLEOPHILIC ADDITIONS TO tert-BUTANESULFINYL IMINES}

Over the past three years, we have extensively explored additions of a number of nucleophiles to tert-butanesulfinyl imines to obtain $\alpha$-branched amines, tertiary carbinamines, $\beta$-amino acids, and 1,2-amino alcohols. 


\section{Asymmetric synthesis of $\alpha$-branched amines}

$\alpha$-Branched amines can be obtained by addition of Grignard reagents to $N$-sulfinyl aldimines in high yields (80-100\%) and with high diastereoselectivities (89:11 to 99:1) [4,7]. Broad substrate generality is observed, with aliphatic and aromatic aldimines and alkyl, aryl, and vinyl Grignards serving as good coupling partners. It is notable that even the addition of Grignard reagents to aldimines having $\alpha$-protons, including the highly acidic arylacetaldimine, proceeds in good yields. To our knowledge, no other organometallic additions to arylacetaldimines have been reported. As shown in eq. 3, a six-membered cyclic transition state with $\mathrm{Mg}$ coordinated to the oxygen of the sulfinyl group is consistent with the sense of induction. The proposed cyclic transition state is also consistent with the reaction proceeding with highest selectivities in noncoordinating solvents.

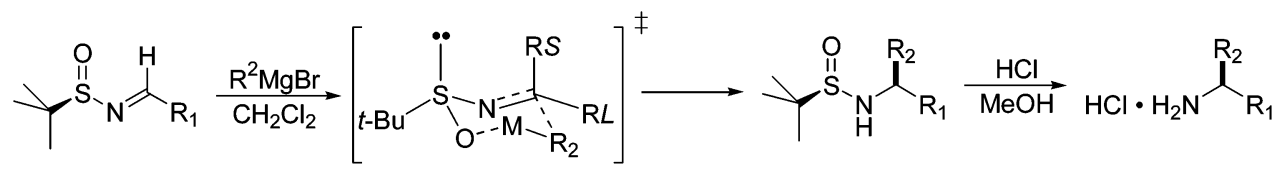

The sulfinyl group is removed from the Grignard addition product by brief treatment with stoichiometric quantities of $\mathrm{HCl}$ in a protic solvent to provide the desired amine hydrochloride in near quantitative yields (eq. 3). Enantiomerically pure material may be obtained by either crystallizing the amine hydrochloride salt or by first chromatographing or recrystallizing the Grignard addition product prior to sulfinyl group removal.

As a second general method for the preparation of $\alpha$-branched amines that complements the Grignard addition chemistry, we have also developed a one-pot asymmetric synthesis of tert-butanesulfinyl protected amines from ketones [8]. In this procedure, ketones are condensed with tert-butanesulfinamide using $\mathrm{Ti}(\mathrm{OEt})_{4}$ followed by addition of $\mathrm{NaBH}_{4}$ to the reaction solution at $-48{ }^{\circ} \mathrm{C}$. The sulfinamide products are obtained in 66-86\% yields for the process and with diastereoselectivities ranging from 90:10 to 97:3 for both aryl alkyl and dialkyl ketones. Notably, Ti(OEt) ${ }_{4}$ not only serves to mediate the imine condensation step, but also serves as a Lewis acid to provide enhanced reduction rates and diastereoselectivities.

\section{Asymmetric synthesis of tertiary carbinamines}

Despite the prevalence of the amine functionality in natural products, synthetic pharmaceuticals, catalysts, and materials, no direct method has yet been reported for the asymmetric synthesis of tertiary carbinamines. To this end, the 1,2-addition of nucleophiles to ketimines has great potential as a general and direct approach. We recently reported the first direct method for the asymmetric synthesis of a broad range of tertiary carbinamines by the unprecedented 1,2-addition of organolithium reagents to sulfinyl ketimines (eq. 4) [5,7]. Significantly, addition of $\mathrm{Me}_{3} \mathrm{Al}$ as a Lewis acid resulted in high yields (85\% average yield) and diastereoselectivities (89:11-99:1) for aryl and alkyl lithium additions to aliphatic and aromatic ketimine substrates. The sense of induction and requirement for noncoordinating solvents is again consistent with a six-membered transition state. Precomplexation of the aluminum reagent to the imine is required, since no reaction occurs if the aluminum reagent is first mixed with the organolithium to form an aluminate species.

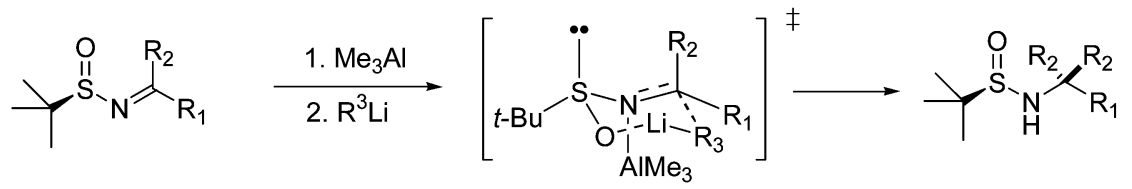


We have also extended this method to the highly efficient synthesis of $N$-protected $\alpha, \alpha$-disubstituted amino acids by the addition of furyllithium to $N$-sulfinyl ketinimines followed by oxidation to the $N$-tert-butanesulfonyl (Bus) $\alpha, \alpha$-disubstituted amino acids (eq. 5) [9].

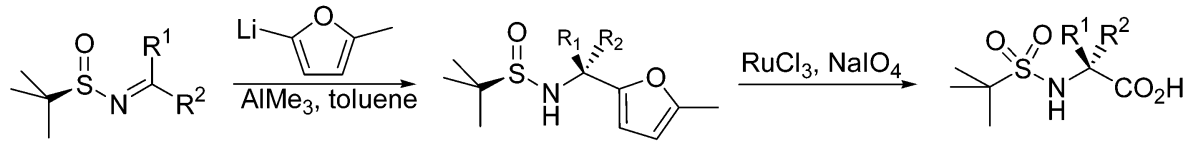

\section{Asymmetric synthesis of highly substituted $\beta$-amino acids}

$\beta$-Amino acids are components of numerous natural products and therapeutic agents. Recently, oligomers of $\beta$-amino acids have received considerable attention owing to their unique structural properties. Although a number of methods are available for the synthesis of $\beta$-substituted amino acids, highly substituted derivatives are not readily accessible. We have found that the addition of titanium enolates to $N$-sulfinyl imines is extremely general and proceeds in high yields and selectivities for the preparation of $\beta$-substituted, $\alpha, \beta$ - and $\beta, \beta$-disubstituted, $\alpha, \beta, \beta$ - and $\alpha, \alpha, \beta$-trisubstituted, and even $\alpha, \alpha, \beta, \beta$-tetrasubstituted $\beta$-amino acid derivatives (eq. 6) [10,11].<smiles>[R]C([R])=NS(=O)C(C)(C)C</smiles>

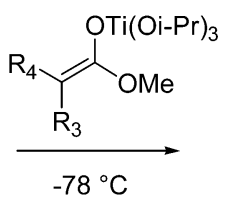<smiles>CCC</smiles>

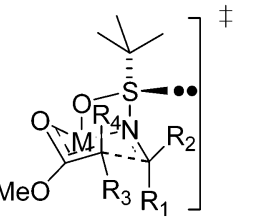

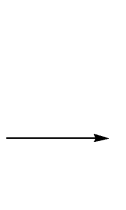<smiles>[R]C([R2])(NS(=O)C(C)(C)C)C([R3])([R])C(=O)OC</smiles>

The tert-butanesulfinyl group not only is an ideal auxiliary for the synthesis of $\beta$-amino esters but also can serve as a versatile, low-molecular-weight protecting group analogous to the Boc protecting group. First, the tert-butanesulfinyl group is stable to basic conditions. For example, $N$-sulfinyl amino esters may be saponified to provide $N$-sulfinyl amino acids. More significantly, the sulfinyl protecting group renders the protected nitrogen non-nucleophilic such that activation and amide bond coupling of the $\beta$-amino acid may be accomplished. The sulfinyl group is then readily cleaved with brief treatment with $\mathrm{HCl}$ in a protic solvent to provide the free amine that may undergo further acylation reactions. These transformations are illustrated in the synthesis of an intermediate to a IIbIIIa antagonist reported by researchers at Monsanto Searle (Fig. 3) [10].

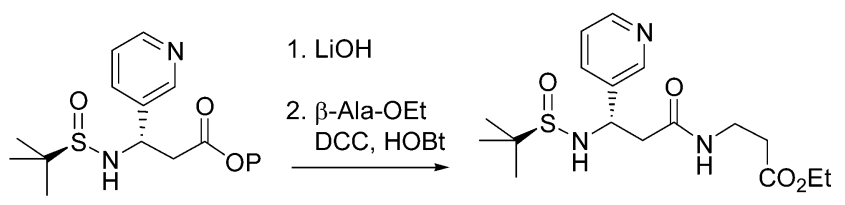

( $85 \%$ for two steps)

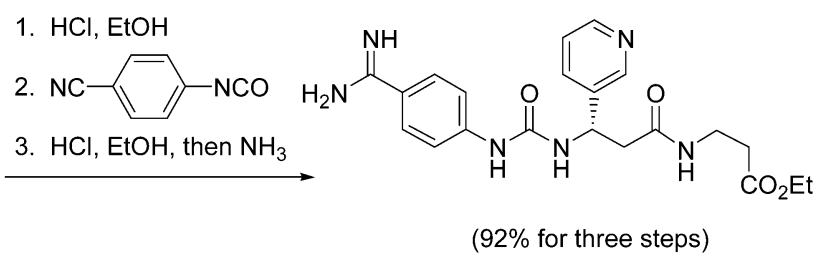

Fig. 3 The tert-butanesulfinyl group serves as a versatile protecting group comparable to the Boc protecting group. 


\section{Asymmetric synthesis of 1,2-amino alcohols}

1,2-Amino alcohols are prevalent in drugs and natural products and serve as important precursors to many chiral ligands for asymmetric catalysis. For this reason, both my group [12] and Barrow and coworkers at Merck [13] have explored the asymmetric synthesis of this compound class by the 1,2 -addition of organometallic reagents to $\alpha$-alkoxy sulfinyl imines. The addition of Grignard reagents to $\alpha$-silyloxy tert-butanesulfinyl aldimines proceeds in high yields (85-96\%) and selectivities (83:17-96:4) in the noncoordinating solvents toluene and $\mathrm{CH}_{2} \mathrm{Cl}_{2}$ (eq. 7). Organometallic reagents may also be added to $\alpha$-benzyloxy sulfinyl aldimines in high yields (80-99 \%) and with good-to-high selectivities (90:10 to 99:1 dr). Interestingly, the sense of induction is opposite to that observed for Grignard reagent additions to sulfinyl aldimines lacking an $\alpha$-coordinating group. Presumably, metal chelation to the $\alpha$-alkoxy group alters the transition state for the reaction.

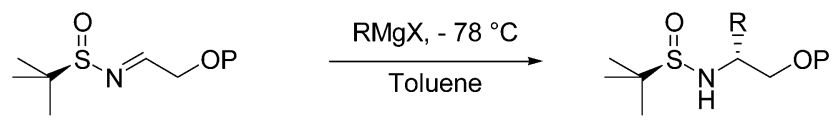

\section{APPLICATION OF tert-BUTANESULFINYL METALLOENAMINES}

Nucleophilic additions to sulfinyl imines have been explored extensively by a number of researchers. In contrast, $\alpha$-deprotonation of sulfinyl imines to provide metalloenamines followed by reaction with electrophiles has only recently been demonstrated in our laboratories [14]. We have determined that metalloenamines react with aldehydes to provide $\beta$-hydroxy $N$-sulfinyl ketimines in high yields and with high diastereoselectivities (eq. 8).

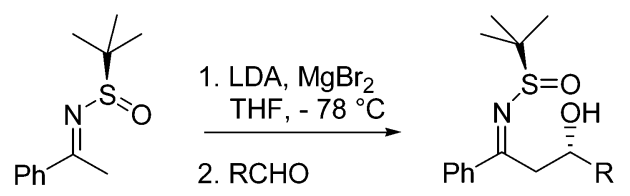

The $\beta$-hydroxy ketimines are versatile intermediates for the preparation of either syn- or anti-1,3-amino alcohols. Reduction with $\mathrm{LiBHEt}_{3}$ provides the anti-1,3 amino alcohol with $\geq 99: 1$ selectivity (eq. 9), while reduction with catecholborane provides the syn-1,3 amino alcohol with $\geq 95: 5$ selectivity (eq. 10). To our knowledge, this is the first method to be reported for the stereoselective synthesis of both the syn- and anti-1,3-amino alcohols from a common synthetic intermediate. The high selectivity results primarily from the tert-butanesulfinyl group because reduction of the hydroxyl epimer of the $\beta$-hydroxy ketimine $\left(\mathrm{R}=\mathrm{Ph}\right.$ ) with $\mathrm{LiBHEt}_{3}$ provided the syn-1,3-amino alchohol with 90:10 diastereoselectivity, while reduction with catecholborane now provided the anti-1,3-amino

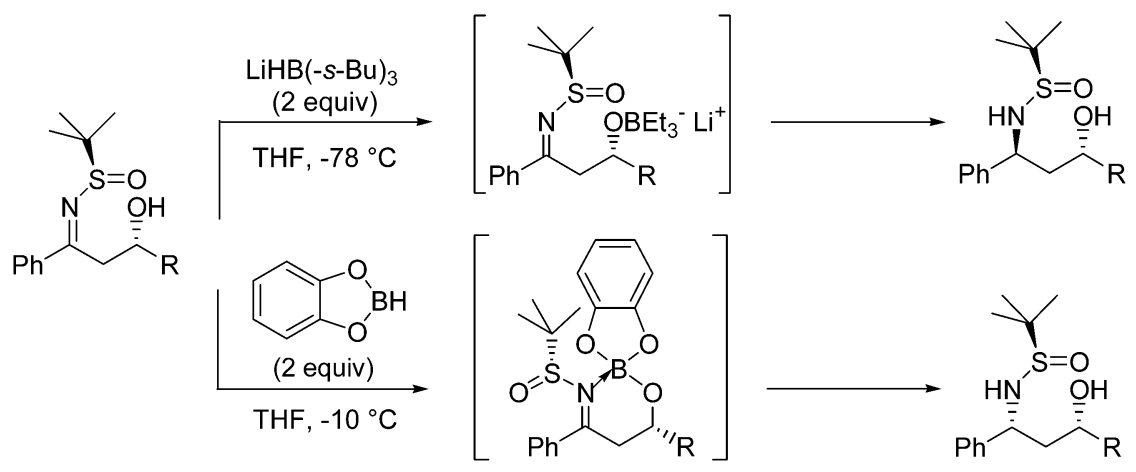


alcohol product with 86:14 diastereoselectivity. The opposite selectivity observed for the reduction with catecholborane relative to $\mathrm{LiBHEt}_{3}$ can be rationalized by considering the geometry of the $N$-sulfinyl imine during the reduction step (eqs. 9 and 10). The $E$-geometry shown for $\beta$-hydroxy $N$-sulfinyl imine is based upon X-ray crystal structural analysis $(\mathrm{R}=\mathrm{Ph})$. The addition of $\mathrm{LiBHEt}_{3}$ is unlikely to change the $N$-sulfinyl imine geometry (eq. 9). In contrast, addition of catecholborane may provide the stable six-membered ring intermediate (eq. 10) in analogy to the stereoselective reduction of $\beta$-hydroxy ketones reported by Evans and Hoveyda [15]. Isomerization from the $E$ - to the Z-imine presumably results in the opposite face selectivity for the catecholborane reduction.

\section{APPLICATION OF A SUPPORT-BOUND tert-BUTANESULFINAMIDE}

Owing to the prevalence of amines in drugs, it is not surprising that the synthesis of compounds that incorporate amines is a major emphasis of combinatorial chemistry efforts. Despite this fact, few methods have been reported for the asymmetric synthesis of libraries of amine-containing compounds. We have, therefore, recently developed an efficient synthesis of a support-bound tert-butanesulfinamide derivative and demonstrated the utility of this linker for the asymmetric synthesis of enantioenriched amines [16]. The support-bound derivative can potentially be applied to the asymmetric synthesis of a range of different bioactive amines. To demonstrate this point, we designed an efficient synthesis of the pavine and isopavine alkaloids (Scheme 1). Condensation of support-bound sulfinamide with $m$-methoxybenzaldehyde is followed by the addition of 3,4-dimethoxybenzyl magnesium bromide. Standard transformations provide the key intermediate I, which upon treatment with $\mathrm{HCl}$ in $\mathrm{CH}_{2} \mathrm{Cl}_{2}$

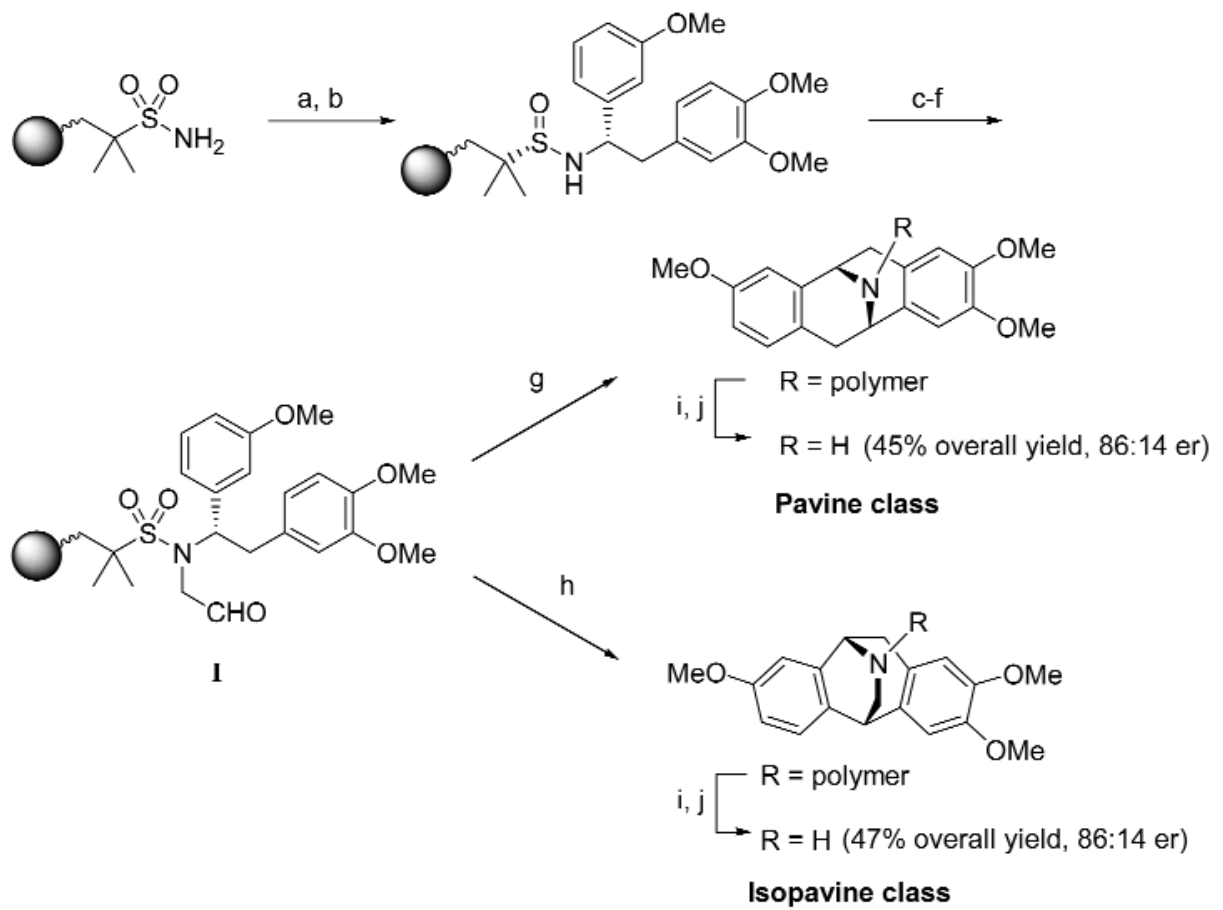

Scheme 1 Asymmetric multistep synthesis of pavine and isopavine alkaloids. Reagents and conditions: (a) 3,4-(MeO) ${ }_{2} \mathrm{PhCH}_{2} \mathrm{CHO}, \mathrm{Ti}(\mathrm{OEt})_{4}$, THF; (b) 3-MeOPhMgBr$, \mathrm{CH}_{2} \mathrm{Cl}_{2},-48{ }^{\circ} \mathrm{C}$; (c) $\mathrm{KO}-t$-Bu, NMP, allyl bromide; (d) $m \mathrm{CPBA}, \mathrm{CH}_{2} \mathrm{Cl}_{2} / \mathrm{DMF}$; (e) $2.5 \% \mathrm{OsO}_{4} / t-\mathrm{BuOH}, \mathrm{NMO}$, THF; (f) $\mathrm{Pb}(\mathrm{OAc})_{4}, 10: 1 \mathrm{CH}_{2} \mathrm{Cl}_{2} / \mathrm{AcOH}$; (g) dil $\mathrm{HCl}, \mathrm{CH}_{2} \mathrm{Cl}_{2}$; (h) 3:1 $\mathrm{CH}_{2} \mathrm{Cl}_{2} / \mathrm{HCO}_{2} \mathrm{H}$; (i) $0.1 \mathrm{~N} \mathrm{TfOH}, 1$,4-dimethoxybenzene, $\mathrm{CH}_{2} \mathrm{Cl}_{2}$; (j) sulfonic acid resin then $\mathrm{NH}_{3} / \mathrm{MeOH}$. 
undergoes two sequential cyclizations to provide the support-bound pavine structure. Alternatively, treatment of I with formic acid provides the support-bound isopavine structure. After release from support, the pavine and the corresponding isopavine products were isolated in 45 and $47 \%$ overall yields, respectively, based upon the starting sulfinamide resin. Notably, only solid-phase extraction with support-bound sulfonic acid followed by elution with ammonia was necessary to provide completely pure material.

\section{CONCLUSION}

Over the past five years, we have developed tert-butanesulfinamide as an extremely versatile chiral amine reagent. tert-Butanesulfinamide is prepared in just two steps from the extremely inexpensive oil waste by-product, tert-butyl disulfide, and directly condenses with aldehydes and ketones to provide tert-butanesulfinyl imines in uniformly high yields. A variety of transformations, such as additions of nucleophiles, may be performed upon the tert-butanesulfinyl imines with high diastereoselectivity and in high yields to provide the desired amine products after straightforward cleavage of the sulfinyl group.

The utility of tert-butanesulfinamide for the asymmetric synthesis of amines is clearly indicated by its extensive use by other researchers. For example, reports have appeared from Olah and Prakash on the highly efficient synthesis of $\alpha$-trifluoromethylamines [17], Davis on the asymmetric synthesis of $\alpha$-amino acids [18], Mabic on the asymmetric synthesis of 1,2-diamines [19], Barrow on the asymmetric synthesis of 1,2-amino alcohols [13], Silverman on novel applications to support-bound $\beta$-amino acids [20], and researchers at Astra Zeneca, Separacor, and Merck on the asymmetric synthesis of $\alpha$-branched amines [21], $\alpha, \alpha$-dibranched amines [22], and $\beta$-amino acids [23] for drug discovery applications.

\section{ACKNOWLEDGMENTS}

This research was supported by the National Science Foundation. The center for New Directions in Organic Synthesis is supported by Bristol-Myers Squibb as a Sponsoring Member and Novartis as a Supporting Member.

\section{REFERENCES}

1. For a more complete overview, see: J. A. Ellman, T. D. Owens, T. P. Tang. Acc. Chem. Res. 35, 984-995 (2002).

2. For leading references on $p$-toluenesulfinamide chemistry, see: (a) P. Zhou, P. B.-C. Chen, F. A. Davis. In Advances in Sulfur Chemistry, C. M. Raynor (Ed.), Vol. 2, pp. 249-282, JAL Press, Stamford, CT (2000); (b) F. A. Davis and R. E. Reddy. Phosphorus, Sulfur Silicon Relat. Elem. 120 \& 121, 291-303 (1997).

3. D. A. Cogan, G. Liu, K. Kim, B. A. Backes, J. A. Ellman. J. Am. Chem. Soc. 20, 8011-8019 (1998).

4. G. Liu, D. A. Cogan, J. A. Ellman. J. Am. Chem. Soc. 119, 9913-9914 (1997).

5. D. A. Cogan and J. A. Ellman. J. Am. Chem. Soc. 121, 268-269 (1999).

6. G. Liu, D. A. Cogan, T. D. Owens, T. P. Tang, J. A. Ellman. J. Org. Chem. 64, 1278-1284 (1999).

7. D. A. Cogan, G. Liu, J. A. Ellman. Tetrahedron 55, 8883-8904 (1999).

8. G. Borg, D. A. Cogan, J. A. Ellman. Tetrahedron Lett. 40, 6709-6712 (1999).

9. G. Borg, M. Chino, J. A. Ellman. Tetrahedron Lett. 42, 1433-1436 (2001).

10. T. P. Tang and J. A. Ellman. J. Org. Chem. 64, 12-13 (1999).

11. T. P. Tang and J. A. Ellman. J. Org. Chem. 67, 7819-7832 (2002).

12. T. P. Tang, S. K. Volkman, J. A. Ellman. J. Org. Chem. 66, 3707-3709 (2001). 
13. J. C. Barrow, P. L. Ngo, J. M. Pellicore, H. G. Selnick, P. G. Nantermet. Tetrahedron Lett. 42, 2051-2054 (2001).

14. T. Kochi, T. P. Tang, J. A. Ellman. J. Am. Chem. Soc. 124, 6518-6519 (2002).

15. D. A. Evans and A. H. Hoveyda. J. Org. Chem. 55, 5190-2 (1990).

16. D. R. Dragoli, M. T. Burdett, J. A. Ellman. J. Am. Chem. Soc. 123, 10127-10128 (2001).

17. (a) G. K. S. Prakash, M. Mandal, G. A. Olah. Angew. Chem. Int. Ed. 40, 589-590 (2001); (b) G. K. S. Prakash, M. Mandal, G. A. Olah. Org. Lett. 3, 2847-2850 (2001); (c) G. K. S. Prakash and M. Mandal. J. Am. Chem. Soc. 124, 6538-6539 (2002).

18. F. A. Davis, S. Lee, H. Zhang, D. L. Fanelli. J. Org. Chem. 65, 8704-8708 (2000).

19. S. Mabic and A. A. Cordi. Tetrahedron 57, 8861-8866 (2001).

20. Y. Lee and R. B. Silverman Org. Lett. 2, 303-306 (2000).

21. (a) N. Plobeck and D. Powell. Tetrahedron Asymmetry 13, 303-310 (2002); (b) D. A. Pflum, D. Krishnamurthy, Z. Han, S. A. Wald, C. H. Senanayake. Tetrahedron Lett. 43, 923-926 (2002).

22. A .W. Shaw and S. J. deSolms. Tetrahedron Lett. 42, 7173-7176 (2001).

23. L. S. Lin, I. E. Kopka, R. A. Mumford, P. A. Magriotis, T. Lanza, Jr., P. L. Durette, T. Kamenecka, D. N. Young, S. E. de Laszlo, E. McCauley, G. Van Riper, U. Kidambi, L. A. Egger, X. Tong, K. Lyons, S. Vincent, R. Stearns, A. Colletti, Y. Teffera, J. Fenyk-Melody, J. A. Schmidt, M. MacCoss, W. K. Hagmann. Bioorg. Med. Chem. Lett. 12, 611-614 (2002). 